\title{
COMPROMISO ORGANIZACIONAL Y PROFESIONAL DEL EQUIPO DE SALUD
}

\author{
COMPROMETIMENTO ORGANIZACIONAL E PROFISSIONAL DA EQUIPE DE SAÚDE \\ *Llapa-Rodríguez, EO, ${ }^{* *}$ Trevizan, MA, ${ }^{* *}$ Shinyashiki, TG, **Mendes Costa, IA. \\ *Enfermera. Profesora de la Universidad Federal de Amazonas. **Enfermera. Profesora \\ Titular de la Escuela de Enfermería de Ribeiráo Preto - Universidad de Sao Paulo / Centro \\ Colaborador de la OMS para el desarrollo de Investigación en Enfermería. Investigador 1 A del \\ CNPq. ***Psicólogo. Profesor Doctor, Facultad de Economía, Administración y Contabilidad de \\ Ribeiráo Preto-Universidad de Sáo Paulo. Brasil.
}

Palabras clave: Recursos humanos; Ética Profesional; Organización y Administración; Servicios de Salud; Motivación

Palavras-chave: Recursos humanos; Ética Profissional; Organizagao e Administragao; Servigos de Saúde; Motivagao.

\section{RESUMEN}

Campo de Estudio: La presente investigación fue realizada en un Servicio de Salud de Atención Primaria en Arequipa-Perú. Objetivo: identificar y analizar el compromiso organizacional y profesional de trabajadores que integran un equipo de atención primaria. Método: Estudio de caso realizado con 12 profesionales de salud, fundamentado en el modelo teórico de Meyer y Allen para el Compromiso Organizacional y Profesional, utilizando entrevistas semi-estructuradas analizadas según análisis de contenido por temática. Resultados: Del análisis resultó la categorización por núcleos temáticos en sus bases Afectiva, Instrumental y Normativa, siendo los seis primeros subtemas relacionados al compromiso organizacional y los cuatro últimos relacionados al compromiso profesional. Conclusiones: Comprendemos que el compromiso contribuye a que los trabajadores se identifiquen con sus roles profesionales y organizacionales, volviéndolos más motivados y por tanto más involucrados. Se espera que organizaciones de salud implementen estrategias y promuevan condiciones que favorezcan el compromiso afectivo entre sus empleados.

\section{RESUMO}

Campo do Estudo: A presente pesquisa foi realizada em uma Unidade de Atencáo Primaria de Arequipa - Perú. Objetivo: identificar e analisar o comprometimento organizacional e profissional de trabalhadores que compóem una equipe de atencáo primaria. Método: Estudo de caso realizado com 12 profissionais da saúde, á luz do modelo teórico de Meyer e Allen para o Comprometimento Organizacional e Profissional, utilizando entrevistas semi-estructuradas. Resultados: Da análise 
resultou a categorizacáo por núcleos temáticos ñas bases Afetiva, Instrumental e Normativa, os seis primeiros relacionados ao comprometimento organizacional, e os quatro últimos relacionados ao comprometimento profissional. Conclusóes: Compreendemos que o comprometimento contribui na identificacáo dos seus trabalhadores com seus papeis profissionais e organizacionais, mostrando-se mais motivados, portanto, mais envolvidos. Espera-se que organizacóes de saúde implementem estrategias, e promovam condicóes que facilitem o comprometimento afetivo nos seus funcionarios.

\section{ABSTRACT}

Scope of study: This research was carried out at a primary health care unit in Arequipa, Peru. Objective: To identify and analyze the organizational and professional commitment of workers in a primary health care team. Method: Case study involving 12 health professionals, in the light of Meyer and Allen's theoretical model for Organizational and Professional Commitment, using semi structured interviews analyzed according to content analysis through subject matter. Results: The analysis led to the categorization by thematic groups on Affective, Instrumental and Normative bases. The first six were related to organizational commitment and the last four to professional commitment. Conclusions: We consider that commitment contributes to the identification of workers with their professional and organizational roles, showing greater motivation and, therefore, more involvement. Health organizations are expected to implement strategies and promote conditions that facilitate affective commitment in their employees.

\section{INTRODUCCIÓN}

Transformaciones sociales y avances tecnológicos invaden el mundo contemporáneo, lo cual cambió la producción y los estilos de vida de las personas. El impacto de estas transformaciones obligó a los trabajadores a pasar por procesos de socialización, como una forma de enfrentar los constantes cambios en su carrera.

Del mismo modo, las instituciones del sector salud no se encuentran fuera de estos cambios inevitables y necesarios. Los profesionales de salud en el cotidiano de su trabajo han asumido actividades más complejas relacionadas con su profesión y organización. De esta forma, estos profesionales enfrentan las divergencias producto de los anhelos de cada sistema en el cual se encuentran inmersos.

Para explicar la situación presentada, diversos estudios ${ }^{(1-4)}$ trataron sobre los conflictos que profesionales del sector salud han enfrentado en su cotidiano, al articularse con los demás sectores institucionales.

En el campo de la salud, el rol de los profesionales ha estado marcado por la racionalidad en el desempeño de sus acciones, exigiendo que estos profesionales cumplan con las normas y reglamentos, explicitados por la burocratización institucional del sector, legado de Taylor. Al ocupar cargos de jefatura, estos profesionales adoptan los supuestos de la gerencia clásica. De esta forma, su compromiso con los valores profesionales se encuentra perjudicado, una vez que su atención se enfoca a las expectativas organizacionales.

Los conflictos producto del compromiso organizacional y profesional han afectado la conducta de los profesionales de la salud. La situación vigente es crítica y estos profesionales están buscando modificar su práctica en los contextos organizacionales.

Además de enfrentar estas disyuntivas, los profesionales durante su desempeño pasan por un conflicto interno, como consecuencia de la bidimensionalidad ${ }^{(3,5)}$ en el compromiso, es decir, además del compromiso con la organización este profesional debe asumir el compromiso con la profesión. En este sentido, la presencia activa de más profesionales en la 
economía sanitaria produjo que ciertas decisiones de los gestores sanitarios fueran vistas como incompatibles con la moralidad profesional ${ }^{(6)}$.

Se observa que las políticas y los proyectos de desarrollo y la gestión de Recursos Humanos (R.H.) han enfocado el comportamiento de las personas en el trabajo, como una forma de favorecer un vínculo más intenso del individuo con las metas organizacionales y profesionales. Este proceso de reestructuración de las fuerzas de trabajo de las instituciones de salud que busca superar el modelo burocrático, tiene implicaciones significativas en las relaciones y vínculos psicológicos, entre profesionales y organizaciones.

En este sentido, consideramos que el compromiso del trabajador, desarrollado en un contexto extremadamente complejo y dinámico como es el caso del área de la salud, se constituye en un tópico de gran interés, adoptando estrategias de solución para la crisis vigente en el sector. Nuestro propósito es contribuir a la aproximación de los valores profesionales con las metas organizacionales. Por tanto, nuestros objetivos son: a) identificar y analizar el compromiso organizacional experimentado por los profesionales que componen un equipo de salud; b) identificar y analizar el compromiso profesional experimentado por los integrantes del equipo de salud.

En esta perspectiva, se presenta el fundamento teórico sobre los compromisos organizacional y profesional.

\section{Fundamento Teórico: Compromisos Organizacional y Profesional}

\section{- Compromiso Organizacional}

Al analizar el compromiso organizacional se encontraron diversos estudios nacionales e internacionales (', los cuales son considerados de relevancia para la comprensión y construcción del compromiso en el trabajo.

Estudios sobre este tópico muestran la importancia de la temática, pues influyen en el bienestar de los trabajadores y en la producción de las organizaciones ${ }^{(8)}$. Gerentes se preocupa y buscan estrategias para que sus empleados estén más motivados y sean un diferencial en su desempeño laboral.

El compromiso puede ser conceptuado de dos formas ${ }^{(14)}$ : (1) cosmopolitan integration, grado en el cual el individuo es activo y se siente parte de los variados niveles de una organización, especialmente en los niveles más altos, (2) organizational introjection, grado en el cual el individuo incorpora una variedad de cualidades y valores organizacionales aprobados.

Otro concepto sobre el compromiso organizacional (15) refleja la estrecha relación entre identificación individual y envolvimiento con la organización. Fue en 1979 cuando comenzaron a desarrollarse investigaciones (12) mencionando que el compromiso estaba también basado en la identificación de antecedentes.

Además de la diversidad de conceptos, varios artículos ${ }^{(16-19)}$ se han preguntado y probado las contribuciones dadas por los diversos autores para entender el compromiso organizacional, lo cual se reflejó al considerar que el compromiso ha sido una variable definida, medida e investigada de forma amplia. Así, Reichers(18) observó esta situación crítica, relacionada con la falta de precisión y redundancia en los conceptos de compromiso, 
y realizó una nueva conceptualización, considerando que el compromiso es una variable que tiene múltiples focos.

Un estudio sobre el estado del arte de investigación en Brasil (20) realizado entre 1996 al 2001, presentó los avances y la importancia del tópico compromiso en el trabajo. La autora enfatiza la necesidad de realizar estudios que integren los diversos focos y bases de este constructo en estudio, utilizando para ello diversas metodologías de investigación, de tal forma que la temática pueda ser de mayor comprensión.

La naturaleza del compromiso en la organización y profesión fue estudiada en base a dos corrientes <5,9,12,18); la actitudinal y la comportamental, también denominadas (21) afectiva e instrumental.

Según los autores citados, el compromiso es una variable multidimensional y posee antecedentes, correlatos y consecuentes que influyen y provocan impacto significativo en sus diversas dimensiones. A manera de ejemplo, se presentan algunos resultados de investigación que correlacionan estas tres variables.

El modelo de antecedentes del compromiso organizacional (22) presenta como variables causales las relaciones: persona - organización, relaciones organizacionales y adaptación personal - organizacional.

Un metaanálisis sobre los antecedentes, correlatos y consecuentes del compromiso organizacional (23) mostró 26 variables clasificadas como antecedentes, 8 como consecuentes y 14 como correlatos.

En el sector salud se encontró el modelo causal de rotación entre enfermeros de hospitales (24), donde se observa que este modelo es afectado por cuatro determinantes: oportunidad, satisfacción en el trabajo, intención de permanecer y entrenamiento. Es decir, cuanto mayor sea la oportunidad en el trabajo, satisfacción y entrenamiento, mayores serán las posibilidades de estos profesionales de permanecer en la institución y, como consecuencia, será reducida su rotación en los servicios hospitalarios.

\section{- Compromiso Profesional}

A pesar de que el compromiso organizacional sea de interés para la mayoría de investigaciones relacionadas con el comportamiento organizacional, se observa al compromiso profesional como una línea de investigación en crecimiento entre profesores e investigadores del área $<5,21,25-27$.

Según el Modelo de los tres Componentes del Compromiso Profesional (28) el individuo comprometido afectivamente es aquel que está ligado emocionalmente con su ocupación. EI individuo comprometido de forma normativa es el que se siente obligado a permanecer en su ocupación y, finalmente, el comprometido instrumentalmente es el individuo que tiene su compromiso vinculado a las pérdidas resultantes si deja su actual ocupación o trabajo.

En un artículo sobre compromiso con la carrera ${ }^{(14)}$ los autores buscaron dirigir y adicionar en el modelo nuevas variables para el compromiso profesional. Los resultados muestran fuertes evidencias de que el involucrarse en el trabajo, el compromiso organizacional y la satisfacción están posiblemente relacionadas con el compromiso con su carrera. 


\section{- El modelo de los tres componentes del compromiso Organizacional y Profesional}

A efectos de esta investigación se presentó la reconceptualización del compromiso organizacional (9) y su validación (29). El modelo de los tres componentes del compromiso organizacional y profesional reúne tres corrientes teóricas: compromiso afectivo, compromiso instrumental y compromiso normativo.

El enfoque afectivo (12) considera al compromiso como: 1. Una fuerte creencia y aceptación de los objetivos y valores de la organización y de la profesión. 2. Buena voluntad para ejercer un esfuerzo considerable en beneficio de la organización y de la profesión. 3. Un fuerte deseo de mantenerse como miembro de la organización y de la profesión.

El enfoque instrumental (13) o del Side bet, menciona al compromiso como "la disposición para estar comprometido en líneas consistentes de actividad". Este enfoque es considerado también como programático y no actitudinal ${ }^{(30)}$, caracterizado por aquel empleado que se encuentra físicamente en su trabajo, pero que no invierte integralmente su capital humano, es decir, permanece no por la existencia de un vínculo emocional, sino porque los costos de no permanecer en él, pueden ser muy altos.

El enfoque normativo conceptualiza al compromiso como la totalidad de presiones normativas internalizadas para trabajar de acuerdo con los objetivos $e$ intereses organizacionales, en donde el individuo cree que es correcto y moral hacerlo. Al mismo tiempo, menciona que este compromiso se establece a través del control ejercido sobre el desempeño del trabajador.

Estos tres enfoques servirán para poder desarrollar y pautar la presente investigación.

\section{METODOLOGÍA}

El estudio fue conducido bajo la óptica de la metodología cualitativa, a través del estudio de caso, considerando que brinda mejores condiciones para vislumbrar y discutir la dimensión subjetiva en la relación organización - individuo - profesión.

La investigación fue realizada en un Servicio de Salud de Atención Primaria perteneciente al Distrito de Cercado, localizado en la ciudad, en el departamento de Arequipa, vinculada al Ministerio de Salud del Perú, que autorizó la realización de la investigación con los integrantes del equipo. Constituyeron las unidades de análisis doce integrantes del equipo multiprofesional incluyendo: tres odontólogos, tres médicos, dos administrativos, una obstetra, una enfermera, una fisioterapeuta y una bióloga.

El proyecto previamente aprobado por el Comité de Ética e Investigación de la Escuela de Enfermería de Ribeirao Preto-USP, viabilizó la recolección de datos. El cuestionario a través de entrevista semi-estructurada (Anexo 1) permitió identificar la vivencia del equipo de salud con relación a su compromiso organizacional y profesional, previo conocimiento y aceptación del término de consentimiento libre e informado por parte de cada uno de los sujetos investigados. De esta forma fueron establecidos horarios adecuados para cada informante; el tiempo para cada entrevista varió entre 40 y 50 minutos. La entrevista permitió un flujo de comunicación interactivo sobre el tópico en estudio.

El instrumento compuesto de dos partes, la primera con preguntas cerradas con el objetivo de buscar informaciones de aspectos socio-demográficos, de formación profesional y del 
trabajo del profesional, la segunda con preguntas abiertas a partir de preguntas guía sobre el compromiso organizacional y profesional, fundamentadas en el modelo teórico de las tres dimensiones de Meyer y Allen.

El procesamiento y análisis de los datos cualitativos fue realizado de forma sistemática con los testimonios de los sujetos participantes, utilizando análisis de contenido por categoría temática (31), identificando y categorizando los núcleos de sentido en base a los tres enfoques del compromiso organizacional y profesional. Por otro lado, el análisis de datos cuantitativos a través del procesamiento con estadística simple según número y frecuencia.

\section{RESULTADOS Y DISCUSIÓN}

En términos del perfil socio-demográfico y profesional, en el grupo estudiado, predominó el sexo femenino $(75 \%)$, casados $(58,33 \%)$, con edades entre 36 a 40 años $(41,67 \%)$ y conyugue de posición en la familia $(41,67 \%)$.

Las características funcionales de los doce participantes indican que $58 \%$ de profesionales de salud trabajan en otras instituciones además del servicio de salud en estudio, siendo 60 meses el tiempo promedio de servicio en esta unidad. Con relación a las características académicas los entrevistados son oriundos de universidades privadas $(75 \%)$, tienen nivel superior completo (75\%), siendo variable el tiempo de formación en años.

Se procedió al análisis y categorización del compromiso organizacional y profesional fundamentado en el modelo de los tres componentes de Meyer y Allen y el Análisis de Contenido. De este análisis resultó la categorización por núcleos temáticos presentados a continuación.

\section{Núcleo Temático - Compromiso Organizacional Afectivo}

De acuerdo con los datos obtenidos este núcleo fue clasificado en cuatro subtemas:

\section{Subtema 1 -Compromiso vinculado al hecho de ser una organización de proyección social}

Este subtema agrupó las respuestas de los profesionales que muestran su compromiso relacionado a la función de proyección social de la unidad básica en estudio, como los testimonios que siguen:

...por labor social (O1) ... porque es una institución médica, con un marcado espíritu de apoyo y medio social... (MD8) ...el trabajo que realizamos es de ayuda al prójimo... (A9)... es una obra de bien social dirigida a la comunidad (O1)

Se observó que el $25 \%$ de los participantes del estudio valoran y se comprometen con su unidad de trabajo, toda vez que ésta se proyecta socialmente. Desde esta perspectiva, el compromiso organizacional de base afectiva representa una fuerte relación del individuo con la organización. Este vínculo se debe principalmente a sentimientos de aceptación de creencias, identificación y asimilación de valores organizacionales que convergen con los objetivos de los trabajadores. 


\section{Subtema 2 - Compromiso considerado como sentimiento de lealtad en retribución a la organización}

Los profesionales mostraron testimonios que evidencian sus sentimientos de lealtad y retribución, como

...lo apoyé en momentos difíciles...siempre intento ver lo mejor para la institución (M12)...creo que trabajo y doy lo mejor para que las cosas salgan bien... (Ob3)...me acogen... por lo tanto, retribuyo y soy leal...(B7).

Múltiples factores dentro y fuera de la organización pueden influir en la adopción de actitudes y comportamiento. En este sentido, la lealtad es vista como parte del perfil del trabajador, siendo una valiosa retribución que la organización desea. Por otro lado, algunos entrevistados mencionaron sentimiento contrarios en relación a la organización:

\footnotetext{
... no valorizan la lealtad... (F5)...no consideran al profesional, no incentivan...no hay futuro (F5).. .siento que estoy mal pagada (O6).
}

En la actualidad, el capital humano es el activo más importante de las organizaciones, y su premisa guía es que las personas asuman su desarrollo profesional en un ambiente que reconozca sus necesidades y valore sus competencias. Si estos empleados encuentran en sus ambientes de trabajo estas condiciones acreditamos que su lealtad será reflejada en sus acciones.

\section{Subtema 3 - Compromiso relacionado al sentimiento de identificación con la organización}

Se resalta que los testimonios están de acuerdo con la definición del compromiso de base afectiva (12). Para estos autores uno de los factores que caracterizan el compromiso es el fundamentado en la afectividad, pues expresa "un fuerte deseo de mantenerse como miembro de la organización". Como queda expresado a continuación por los participantes:

...me gusta mi ambiente de trabajo...(B7)...por el carisma... me siento identificado... porque trabajo en ella y la vi nacer (M12)... me siento parte, y cada vez más, me integro a su estructura (MD8).

Por otro lado se encontró un testimonio que caracteriza un trabajador desalentado, no sintiéndose parte de la organización.

no me consideran.entonces no pertenezco a esta organización. Me siento excluida... (O2)

El sentimiento de este trabajador denota que se encuentra desmotivado, no sintiéndose parte de la organización. Estudio sobre satisfacción laboral (32) menciona que las variables tensión laboral y promoción profesional son las menos valoradas. Es necesario resaltar que este profesional no tiene vínculo con la organización, es uno de los trabajadores por servicios no personales, lo que podría estar influenciando su respuesta. 
Subtema 4 - Compromiso con la organización con miras al desempeño de la vocación profesional

Los testimonios expresan que para estos trabajadores, la organización es un canal o un medio, el cual es utilizado para poder cumplir con la vocación profesional, como expresan a continuación:

...porque permite realizarme como ser humano y cumplir con mi vocación ... $($ MD8)... me permite llegar más al paciente...ayudarlo (M10)... dentro de mi formación...los pilares más importantes son el servicio a los demás y aquí puedo hacerlo (A9).

Entendemos que los objetivos y valores profesionales de los empleados son utilizados para comprometerse con la organización, lo que nos permite inferir que para ellos, aparentemente, la profesión tiene primacía sobre la organización.

\section{Núcleo Temático - Compromiso Organizacional Instrumental}

En este núcleo, nuestros resultados se vincularon a un único subtema que se presenta a continuación.

\section{Subtema 1 - Compromiso vinculado a la necesidad de trabajo del individuo}

Esta categoría refleja el sentimiento de los profesionales, al expresar su compromiso con la organización fundamentado en la propia necesidad de trabajo. Los testimonios indican la necesidad económica y la falta de oportunidad de empleo en el medio en donde se desarrollan.

Se presentó la oportunidad y la necesidad... en estos tiempos... (A11). Porque no había otro lugar de trabajo (F5)...con la situación actual...en algunos lugares, uno deja el currículo...nadie te llama, lo digo por experiencia propia (F5) Viendo la situación sin alternativas... (E4)

En un mundo globalizado, las causas de crisis pueden ser incontables y aun la recesión económica propia del país son condiciones que propician la disminución de oportunidades de empleo y dificultades para que el trabajador se introduzca dentro del campo de trabajo.

\section{Núcleo Temático - Compromiso Organizacional Normativo}

También en este núcleo, nuestros datos fueron incluidos en un subtema.

\section{Subtema 1 - Compromiso por motivación y envolvimiento moral}

Como se observa a continuación, esta categoría presenta dos trabajadores con compromiso relacionado con la motivación, dos se comprometen con la organización a través del vínculo moral y uno tiene compromiso fundamentado en las dimensiones de motivación y moral. Esta categoría fue construida basada en los testimonios que siguen:

\footnotetext{
Me invitaron a trabajar en esta institución (O2), por unión familiar (E4), ...debido al proyecto elaborado conjuntamente con el gestor (O6)... porque es una institución católica (A11)...tengo mi formación religiosa, estudié en un colegio parroquial, lo cual me motiva... (M10).
} 
Podemos inferir que para cada persona existe un grupo de necesidades y fuerzas internas que dinamizan e impulsan sus comportamientos e actitudes, los que son dirigidos para algo observable, lo que se evidencia frente a las diversas circunstancias en su cotidiano.

\section{Núcleo Temático - Compromiso Profesional Afectivo}

Según referencial teórico ${ }^{(3)}$, el compromiso profesional se desarrolla gradualmente transformándose durante la carrera del trabajador. Para este primer núcleo temático fueron elaborados los siguientes subtemas, basados en los testimonios de los participantes del estudio.

\section{Subtema 1 - Compromiso profesional vinculado con la vocación}

Se encontró que del total de sujetos investigados, 91,7\% presentan compromiso profesional fundamentado en la afectividad. Los testimonios lo demuestran:

...Por vocación...servir al prójimo y la comunidad...esto es ser enfermera
(E4)...cumplir con mi vocación, la cual siempre tuve (MD8)...fui formado para esto, es
lo único que sé hacer y lo hago bien (M12)...permite realizarme ... y cumplir con mi
vocación..es parte de mi vida (MD8).. jamás me arrepentí (M10) Porque me gusta,
me encanta lo que hago (F5) Soy feliz haciendo lo que hago (A9)... quiero inclusive
que mis hijas sigan mi profesión (O2).

La vocación es considerada una aptitud necesaria para que el profesional se desempeñe dentro de su cotidiano, ofreciendo satisfacción y deseo de permanecer en su ocupación. Por otro lado, de acuerdo con nuestra comprensión acreditamos que el testimonio $\mathrm{O} 2$ direccionó de forma inadecuada su vocación, al mencionar que desea que también sus hijas continúen con su profesión.

\section{Subtema 2 - Compromiso profesional relacionado a la intención de continuar en la profesión}

La intención de los sujetos de no dejar su actual profesión evidencia la naturaleza del vínculo afectivo-actitudinal para con la misma.

Si, escogería.es una profesión en la que puedo trabajar hasta cuando quiera (O2), escogería la misma (Ob3), sí, sin pensar (F5)

Estas afirmaciones se caracterizan por una fuerte creencia y aceptación de valores y objetivos para con su profesión, mencionado por Meyer y Allen en su dimensión afectiva.

\section{Subtema 3 - Compromiso profesional en busca de la autorrealización}

En su mayoría, los testimonios expresados muestran sentimientos de realización tanto profesional como personal. La necesidad de auto-realización es tan importante para poder ejecutar sus actividades diarias, es aquello que da sentido a su vida e impulsa su caminar durante la construcción de su propia vida profesional. 
.. me siento contenta, me siento desarrollada en $\mathrm{mi}$ carrera, realizada (Ob3),...me permite realizarme como ser humano... (MD8)...la profesión ofrece grandes satisfacciones (M10)

Los testimonios expresan claramente el sentimiento de realización vinculado al compromiso profesional, a través de estos podemos también observar que además de la realización profesional existe el sentimiento de realización personal.

\section{Núcleo Temático -Compromiso Profesional Normativo}

Nuestros datos frente a este núcleo se vincularon a un subtema.

\section{Subtema 1 - Compromiso profesional frente a las presiones normativas internalizadas}

Los resultados muestran la carga de presiones normativas internalizadas por los profesionales, aspecto que los predispone a comprometerse con su profesión al incorporar los objetivos y metas. Los participantes expresaron:

...mis padres me incentivaron... no tenía la menor idea...sabía que era curar dientes nada más (01)... servir al prójimo y a la comunicad, esto es ser enfermera (E4)... cumplir con mi vocación, la cual siempre tuve. (MD8).hice una promesa a Dios de atender a las personas...(F5).

Observamos que estos participantes inicialmente se dejaron influenciar y optaron por su profesión sin mucha convicción, en el momento de decidir; sin embargo, con el tiempo su visión sobre su profesión, para muchos de ellos, cambió.

Los profesionales en estudio expresaron a través de sus testimonios sus formas de compromiso sea este con su profesión u organización, o con ambas, aspectos que los aproximaron o alejaron de sus valores profesionales y metas organizacionales.

\section{CONSIDERACIONES FINALES}

Considerando que el compromiso es fundamental para la introducción del trabajador en la organización, así como para el desarrollo de sus actividades, los profesionales del equipo de salud participantes de este estudio indicaron las formas como visionan y viven su compromiso organizacional y profesional a partir de su experiencia frente al reto de trabajar en una unidad básica de salud, en donde enfrentan la bidimensionalidad frente a su compromiso organizacional y profesional.

Comprendemos que el compromiso contribuye a que los trabajadores se identifiquen con sus roles profesionales y con la organización de trabajo, volviéndose más motivados y por tanto más involucrados.

Los resultados encontrados a partir del análisis y las características de las respuestas de los doce sujetos participantes permitieron la identificación de los núcleos temáticos fundamentados en el modelo de los tres componentes de Meyer y Allen, es decir, en sus diversos enfoques: afectivo, instrumental y normativo

El Compromiso Profesional Afectivo fue predominante en los profesionales de salud, lo que se evidencia cuando mencionan sentirse contentos, realizados, felices y satisfechos con su 
profesión. Por otro lado, el Compromiso Organizacional en su mayoría fue vinculado a la necesidad de trabajo del individuo y a presiones normativas internas.

Una de las contribuciones que podemos extraer de este estudio es pensar y ver el compromiso como un valor que debe ser parte del perfil del profesional de salud. Los resultados de esta investigación de acuerdo con nuestra comprensión, permiten vislumbrar estrategias para la utilización de recursos humanos en el sector salud, invirtiendo en la gestión de talentos, propiciando de esta forma una mejor atención brindada al cliente interno y externo.

Partiendo del supuesto de que el compromiso es fundamental para la introducción del trabajador en su profesión y organización, la conciliación de los dos tipos de compromisos, en el cotidiano de las organizaciones, favorece el alcance de los propósitos y objetivos institucionales y profesionales, y como producto se obtendrá una mayor eficiencia y eficacia del profesional.

Es fundamental favorecer y estimular el desarrollo del compromiso afectivo entre los trabajadores debido a sus consecuencias positivas tanto para la organización como para la profesión. El empleado con compromiso afectivo se vuelve un recurso valioso y singular para el éxito de la organización.

Es necesario resaltar que las facultades y escuelas que forman profesionales de salud deben estar atentas a la importancia de este tópico y alertas sobre la escasez bibliográfica relacionando estos dos focos de compromiso en el área de la salud, considerando que esta temática debe ser comprendida, incorporada y aplicada por los administradores y gerentes de salud como un valor esencial de la profesión y organización.

\section{REFERENCIAS}

1. FERNANDES, M. S. A fungáo do enfermeiro nos anos 90: replica de um estudo. 2000.134 p. Dissertagáo (Mestrado em Enfermagem) - Escola de Enfermagem de Ribeiráo Preto, Universidade de Sáo Paulo, Ribeiráo Preto, 2000.

2. FERRAZ, C. A. A transfiguracáo da administracáo em enfermagem: da gerencia científica á gerencia sensível. 1995. 248 p. Tese (Doutorado em Enfermagem) - Escola de Enfermagem de Ribeiráo Preto, Universidade de Sáo Paulo, Ribeiráo Preto, 1995.

3. TREVIZAN, M.. A. Enfermagem hospitalar: administragáo \& burocracia, Brasilia: Editora Universidade de Brasilia, 1988, $142 \mathrm{p}$.

4. FERREIRA- SANTOS, C. A. A enfermagem como profissáo. Sáo Paulo: Livraria Pionera Editora,. 1973. 176 p.

5. SCHEIBLE. A.C.F. Comprometimento no trabalho : um estudo de caso de suas relacóes com desempenho e práticas de gestáo. 2004. 134 f. Dissertagáo (Mestrado em administragáo de empresas)- Escola de administragáo, Universidade Federal da Bahia, Salvador, 2004.

6. GARCIA, D . Professional Ethic and Institutional Ethic: Convergency or Conflict. Revista Española de Salud Pública, v. 80, n. 5, p. 457-467, 2006.

7. BASTOS, A.ntonioVirgilio Bittencourt; BORGES-ANDRADE, Jairo Eduardo. Comprometimento com o trabalho: Padróes em diferentes contextos organizacionais. Revista de Administracáo de Empresas, Sáo Paulo, v. 42, n. 2, p. 31-41, Abr./Jun. 2002. 8. MEYER,J.P; HERSCOVITCH,L. Commitment in the workplace : toward a general model. Human resource management review, New Orleans, v.11, p. 299-326, 2001. 
9. MEYER, J.P; ALLEN, N.J. A three-component conceptualization of organizational commitment. Human resource management review, New Orleans, 1, n.1, p. 61-89, 1991.

10. RANDALL, D. Commitment and the organization: the organization man revisited. Academy of management review, United States, v.12, n. 3, p. 460-471, 1987.

11. WIENER, Y. Commitment in organizations : a normative view. Academy of management review, United States, v.7, n.3, p. 418-428, Jul.1982.

12. MOWDAY ,R. T; STEERS, R.M; PORTER, L. W. The measurement of organizational commitment. Journal of vocational behavior, San Diego, v.14, p.224-247, 1979.

13. BECKER, H.S. Notes on the concept of commitment. The Americam Journal Sociology. Chicago, v. 66, n. 1,p. 32-40. 1960.

14. GOULDER, H. P. Dimensions of organizational commitment. Adminstrative science quarterly, v. 4, p. 468-490, 1960.

15. STEERS, R. M. Antecedents and outcomes of organizational commitment. Administative Science Quarterly, New York, v. 22, p. 46-56, 1977.

16. BATEMAN, T. S; STRASSER, S. A longitudinal analysis of the antecedents of organizational commitment". Academy of Management Journal, United State, v. 27, n. 1, p. 95-112. Mar. 1984.

17. DE COTTIS, T.A; SUMMERS, T.P.A. A path analysis of a model of the antecedents and consequences of organizational commitment. Human Relations, London, v. 40, n. 7, p. 445 470, 1987.

18. REICHERS, A. A review and reconceptualization of organizational commitment. Academy of management review, United States, v.10, n.3, p. 465-476, 1985.

19. SCOTTER, J.R.V. Relationships of task performance and contextual performance with turnover, job satisfaction an affective commitment. Human resource management review, New Orleans, v.10, n. 1, p. 79-95, 2000.

20. DEMO, G. Comprometimento no trabalho: uma síntese do estado da arte e uma revisáo da produgáo nacional. Revista Psicologia: Organizacóes e Trabalho, Florianópolis, v.3, n. 2, p. 185-213, Jul./Dez.2003.

21. BASTOS, A.V. B. Comprometimentos no trabalho : a estrutura dos vínculos do trabalhador com a organizacáo, a carreira e o sindicato, 1994. 294 p.Tese (Doutorado em Psicologia) - Instituto de Psicologia, Universidade de Brasilia, Brasilia, 1994.

22. LUTHANS, F.; BAACK, D.; TAYLOR, L. Organizational commitment: analysis of antecedents. Human relations, v. 40, n. 4, p. 219-236, 1987.

23. MATHIEU, J. E; ZAJAC, D.M. A review and Meta-Analysis of the Antecedenst, Correlates and Consequences of Organizational commitment. Psychological Bulletin,v.180, n. 2, p. 171-194, Sep. 1990.

24. PRICE,J. L; MUELLER,C.W. A causal model of turnover for nurses. Academy of management journal, United States, v. 24, n. 3, p. 543-565, 1981

25. BLAU, G. Testing for a four- dimensional structure of occupational commitment. Journal of Occupational and Organizational Psychology, United Kingdom, v. 76, p. 469-488. 2003.

26. GOULET, L.R; SINGH, P. Career commitment : a reexamination and an extension. Journal of vocational behavior, United States, v.61, p. 73-91, 2002.

27. WALLACE, J.E. Professional and organizational commitment : compatible or incompatible. Journal of vocational behavior, San Diego, v. 42, p. 333-349, 1993.

28. MEYER,J.P; ALLEN, N.J; SMITH, C.A. Commitment to organizations and occupations : extension and test of a three-components conceptualization. Journal of applied psychology, Marylands, v. 78, n. 4, p. 538-551, 1993.

29. MEDEIROS, C.A.F.; ENDERS, W.T. Validagáo do Modelo de Conceitualizagáo de Tres Componentes do Comprometimento Organizacional (Meyer e Allen, 1991). Revista de Administracáo Contemporánea, Curitiba, v. 2, n. 3, p. 67-87, Set./Dez. 1988 
30. DAVENPORT, T.O. O Capital Humano: o que é e porque as pessoas investem nele. Sao Paulo. Nobel 2001. 248 p.

31. BARDIN, L. Análise de conteúdo.1`. Ed. Lisboa: Edigóes 70 Ltda, 1977, 225 p.

32. SAN MARTÍN, M.I.F. et al. Satisfacción Laboral de los Profesionales de Atención Primaria del área 10 del insalud de madrid. Revista Española de Salud Pública, Madrid, v. 74, n. 2, p. 139-147, Mar./Abr. 2000.

33. BASTOS, A.V.B. Padröes de comprometimento com a profissáo e a organizagáo: o impacto de fatores pessoais e da natureza do trabalho. Revista de Administragáo, Sáo Paulo, v. 35, n. 4, p. 48-60, Oct./Dez. 2000. 
ANEXO 1

UNIVERSIDADE DE SAO PAULO

ESCOLA DE ENFERMAGEM DE RIBEIRÁO PRETO

PESQUISA SOBRE COMPROMISSO

PARTE I

DADOS PESSOAIS

\begin{tabular}{|c|c|c|c|c|}
\hline \multicolumn{5}{|c|}{ PROFISSĀO DO PARTICIPANTE: } \\
\hline $\begin{array}{l}\text { IDADE: } \\
\square \text { menos de } 25 \mathrm{~A} \\
\square \text { DE } 26 \text { A } 30 \\
\square \text { DE } 31 \text { A } 35 \\
\square \text { DE } 36 \text { A } 40 \\
\square \text { DE } 41 \text { A } 45 \\
\square \text { DE } 46 \text { A } 50 \\
\square \text { mais de } 50 \text { A. }\end{array}$ & $\begin{array}{l}\text { SEXO: } \\
\square \text { Masc } \\
\square \text { Fem. }\end{array}$ & $\begin{array}{l}\text { ESTADO } \\
\text { CIVIL: } \\
\square \text { SOLTEIRO } \\
\square \text { CASADO } \\
\square \text { VIUUVO } \\
\square \text { DIVORC. } \\
\square \text { OUTRO }\end{array}$ & $\begin{array}{l}\text { ESCOLARIDADE: } \\
\square \text { SUPERIOR } \\
\text { COMPLETA } \\
\square \text { MESTRADO } \\
\square \text { DOUTORADO } \\
\square \text { ESPECIALIDADE } \\
\square \text { OUTROS }\end{array}$ & $\begin{array}{l}\text { POSICCÃO NA } \\
\text { FAMÍLIA: } \\
\square \text { CHEFE DE } \\
\text { FAMILIA } \\
\square \text { CÔNJUGE } \\
\square \text { FILHO(A) } \\
\square \text { PARENTE }\end{array}$ \\
\hline
\end{tabular}

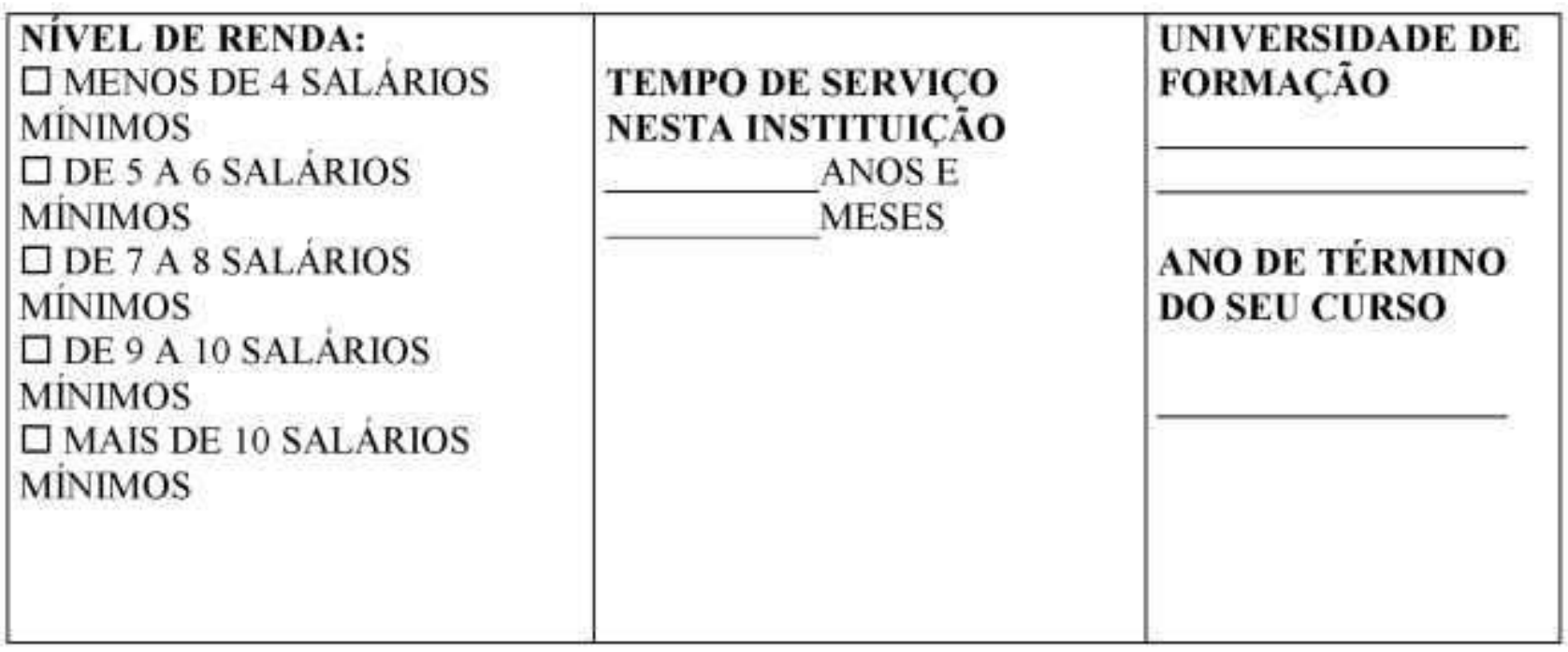




\section{PESQUISA SOBRE COMPROMETIMENTO}

O levantamento que estamos realizando no seu servico visa compreender como o trabalhador se relaciona com varios aspectos da sua vida no trabalho.

Nossa preocupacäo origina-se no interesse em conhecer melhor como o trabalhador está percebendo e evaluando as mudancas que estäo ocorrendo.

Trata-se de uma pesquisa da Universidade de Säo Paulo. Assim, os dados e resultados seräo analisados fora da organizacäo. Por este motivo, a informacäo será mantida sob o mais absoluto sigilo. Esperamos contar com sua disponibilidade e que se sintam confiantes a conversar conosco.

A seguir, encontrará uma série de perguntas abertas que representam suas possíveis opiniöes com relacäo ao tema: comprometimento organizacional e professional.

\section{PARTE II}

\section{COMPROMETIMENTO COM A ORGANIZACÄO}

1. Por que escolheu trabalhar nesta instituicäo?

2. Se pudesse escolher novamente, escolheria trabalhar nesta instituicäo? Por qué?

3. Vocé se sente parte desta instituicäo?

4. Considera que esta organizacäo merece sua lealdade? Por qué?

5. Vocé acha que teria poucas alternativas de trabalho se deixasse esta instituicäo agora?

\section{COMPROMETIMENTO COM A PROFISSÄO}

1. Por que escolheu sua atual profissäo?

2. Na sua opiniäo, sua profissäo é ideal para trabalhar o resto da sua vida?

3. Se pudesse escolher novamente, escolheria a mesma profissäo? Por qué? 
